\title{
Patients with Vitamin D Deficiency Are at Higher Risk of Developing Calcified and Mixed Plaques
}

\author{
(1) Ferhat Eyyüpkoca1, (1) Yasin Yüksel2, (1) Mehmet Sait Altıntaş3, (1) Onur Yıldırım3 \\ (D) Ajar Koçak1
}

${ }^{1}$ Sincan State Hospital, Clinic of Cardiology, Ankara, Turkey

2İstanbul Training and Research Hospital, Clinic of Cardiology, İstanbul, Turkey

3İstanbul Yedikule Training and Research Hospital, Clinic of Cardiology, İstanbul, Turkey

\begin{abstract}
Objectives: Vitamin D plays a role in the cardiovascular system through its pleomorphic effects. In some studies, it has been reported that the relationship between vitamin D deficiency and coronary artery calcification is inconsistent. In this study, it was aimed to evaluate the relationship between the vitamin D level and coronary artery calcium score (CACS), plaque presence, and plaque type.

Materials and Methods: Included in this retrospective cohort study were 719 patients who had no previously known coronary artery disease (CVD), and for whom coronary computed tomography angiography (CCTA) was performed between 2015 and 2019. Patients were classified as normal, inadequate, or deficient according to their levels of vitamin $\mathrm{D}$ deficiency. They were evaluated according to the presence of plaque on their CCTA or CACS $>0$ atherosclerosis. Moreover, patients were separated into four groups, comprising zero-plaque (those that were not plaque according to the plaque type), mere fatty plaque (CACS=0), mere calcified plaque, and mixed plaque. Age, sex, smoking status, diabetes mellitus, hypertension, and hyperlipidemia were evaluated as traditional risk factors.
\end{abstract}

Results: In $18.4 \%$ of the patients, the vitamin D levels were normal, whereas they were inadequate in $65 \%$ and deficient in $16.7 \%$. The median CACS of the patients was 0 (range: $0-3759$ ), and mere fatty plaque was found in $13.5 \%$ of patients,

Address for Correspondence: Ferhat Eyyüpkoca, Sincan State Hospital, Clinic of Cardiology, Ankara, Turkey e-mail: eyupkocaferhat@gmail.com ORCID: orcid.org/0000-0003-2630-3619

Received: 31.03.2021 Accepted: 31.08 .2021

Cite this article as: Eyyüpkoca F, Yüksel Y, Altıntaş MS, Yıldırım O, Koçak A. Patients with Vitamin D Deficiency Are at Higher Risk of Developing Calcified and Mixed Plaques. EJCM 2021;9(3):158-168.

DOI: 10.32596/ejcm.galenos.2021-03-024 
whereas $13.4 \%$ had mere calcified plaque, and $27.5 \%$ had mixed plaque. A negative correlation was detected between the vitamin D levels and CACSs $(\mathrm{r}=0.345 ; \mathrm{p}<0.001)$. The median CACS in those with vitamin $\mathrm{D}$ deficiency was higher when compared to those with inadequate and normal levels (normal: 0 vs inadequate: 0 vs deficient: $7 ; p<0.001$ ). Regardless of the traditional risk factors, vitamin D deficiency was found to be an independent predictor of atherosclerosis [odds ratio (OR): 6.9; 95\% confidence interval (CI): 3.53-13.52; $\mathrm{p}<0.001$ ], fatty plaque (OR: 3.04; 95\% CI: 1.34-6.87; $\mathrm{p}=0.008$ ), mere calcified plaque (OR: 13.11; 95\% CI: 3.53-13.52; $\mathrm{p}<0.001$ ), and mixed plaque (OR: 14.27 ; 95\% CI: 5.58-36.50; $\mathrm{p}<0.001)$. Moreover, regardless of the traditional risk factors, the vitamin D deficiency increased the risk of fatty plaque development by 2.37 times in patients with CACS: 0 (OR: 2.37; 95\% CI: 1.01-5.62; $\mathrm{p}=0.045$ ).

Conclusion: A decrease in vitamin D level is associated with an increase in the CACS, and the development of calcified and mixed plaque is more likely when there is vitamin D deficiency. Depending on the incidence of CVDs and vitamin D deficiency in asymptomatic patients, vitamin D supplements can be beneficial.

Keywords: Vitamin D, coronary artery calcium score, atherosclerosis, coronary artery disease

\section{Introduction}

Vitamin D deficiency, which is a major public health problem worldwide, has been shown to be an important risk factor in cardiovascular diseases ${ }^{(1,2)}$. In cardiovascular diseases, coronary artery disease (CAD) is one of the leading causes of morbidity and mortality. Growing evidence has suggested that many factors, including vitamin $\mathrm{D}$, play a role in coronary plaque formation ${ }^{(2-4)}$. Vitamin $\mathrm{D}$ deficiency affects a large number of cells involved in atherogenesis (such as immune cells, endothelial cells, smooth muscle cells, and cardiomyocytes $)^{(5-8)}$.

Coronary artery calcification (CAC) is the pathognomonic finding of atherosclerosis, as well as a good marker of atherosclerotic plaque load ${ }^{(9,10)}$. Moreover, coronary calcium measurements have been associated with histological measurements of atheromatous plaque $^{(11,12)}$. The growing evidence supports the role that vitamin D plays in the development of cardiovascular impacts and CAC; however, there have also been studies that have shown no relationship between them ${ }^{(13-18)}$. Different geography and patient groups form the basis of this contrast. However, no studies that have assessed the relationship between the vitamin D level and the coronary artery calcium score (CACS), presence of plaque, and plaque-type in a wide cohort could be found.
In this study, it was aimed to examine the role of vitamin D deficiency by evaluating the relationship between vitamin D levels and the CACS, presence of plaque, and plaque type.

\section{Materials and Methods}

\section{Study Population}

This retrospective cohort study was designed and undertaken at the Cardiology and Thoracic Surgery Clinics of the İstanbul Yedikule Training and Research Hospital. All aspects of the research were carefully designed to comply with the 2013 Declaration of Helsinki, as well as the principles of good clinical practices. The relevant ethics committee granted approval of the study [İstanbul Training and Research Hospital, University of Health Sciences, Clinical Research Ethics Committee (decision date/no: 24.07.2020/2481)]. Furthermore, the consent of all participants was also obtained in both verbal and written form before the study began.

Included in the study were 719 patients who had no previously known CAD, and for whom coronary computed tomography angiography (CCTA) was performed between 2015 and 2019. The exclusion criteria of the study included the presence of cardiac failure, congenital heart 
disease, history of asthma, and history of CVD, history of pulmonary embolism, chronic obstructive lung disease, and history of kidney disease.

The demographic data (gender, age, hypertension, diabetes mellitus (DM), hyperlipidemia, and smoking status), the laboratory data, and the CCTA data of all participants were obtained from their patient files using the electronic information system of the hospital.

\section{Laboratory Testing}

Results of the blood sample tests were obtained from the patient files as described above. Platelets were measured using the impedance method, and other hemogram parameters were measured using a Sysmex XE 2100 hematology analyzer (Roche Diagnostic, Corp. IN, USA), and hemoglobin was measured photometrically. C-reactive protein was measured using the immunoturbidimetric method, albumin was measured using the bromine cresol green method, triglycerides and total cholesterol were measured using the enzymatic colorimetric method, and high-density lipoprotein (HDL) cholesterol was measured using the homogeneous enzymatic colorimetric method in a Beckman Coulter DX1800 Analyzer (Indianapolis, USA). Low-density lipoprotein (LDL) cholesterol was calculated according to the Friedewald formula.

Vitamin D levels were measured using the $25(\mathrm{OH})$ 2D3 radioimmunoassay method (Beckman Coulter, Indianapolis, USA) in an auto analyzer. Vitamin D was classified as normal (vitamin $\mathrm{D}>30 \mathrm{ng} / \mathrm{mL}$ ), insufficient (10-30 ng/mL), or deficient $(<10 \mathrm{ng} / \mathrm{mL})^{(19)}$.

\section{Coronary Artery Calcification Assessment}

All imaging was performed with a 64 multi-slice computed tomography (Toshiba Aquillon, Japan). During the examination, the heart was scanned in the craniocaudal direction, from the carina to the apex. During the process, imaging was performed using the parameters $120 \mathrm{Kvp}$, $300 \mathrm{~mA}, 75 \mathrm{mAs}$, and 3-mm section thickness. Next, all of the images were transferred to the workstation for calcium scoring and evaluated using a Toshiba Aqua 4.1 device
(Otawara, Japan). The CACS was calculated considering a threshold of $130 \mathrm{HU}$, as described by Agatston et al. ${ }^{(20)}$. A CACS: 0 was evaluated as the absence of CAC. The CACS was categorized into the following five classes: 0 , $1-99,100-399,400-999$ and $\geq 1000$. In all of the coronary segments, the coronary plaque was defined as 1) zeroplaque, 2) calcified (a more intense computed tomography (CT) density than the coronary lumen filled by contrast), 3) non-calcified (less density than the coronary lumen filled by contrast, but more CT density than the connective tissue around it), or 4) mixed (plaque containing both calcified and non-calcified components). One coronary plaque was assigned per coronary segment.

\section{Statistical Analysis}

IBM SPSS Statistics for Windows 20.0 (IBM Corp., Armonk, NY, USA) was used for all of the statistical analyses. To determine whether or not the data were normally distributed, the Kolmogorov-Smirnov test was applied. While the numerical variables were given as the mean \pm standard deviation or median (minimummaximum), the categorical values were given as numbers and percentages. The chi-square test and Fisher exact test were applied to compare the categorical data. The student t-test or Mann-Whitney U test was used in the comparison of numerical variables in two groups according to the normality of the distribution. The ANOVA test (post hoc: Bonferroni test) or Kruskal-Wallis $\mathrm{H}$ test (post hoc: Dunn test) was used to compare the numerical variables between the plaque types according to the normality of the distribution. The Spearman correlation analysis was used for the relationship between the CACS and the vitamin D level. Age, gender, smoking status, DM, hypertension, and hyperlipidemia were considered as traditional risk factors. The association of vitamin D deficiency and the presence of plaque was used in the logistic regression analysis and adjusted traditional risk factors. $\mathrm{P}<0.05$ was considered as statistically significant. 


\section{Results}

The mean age of patients was $51.9 \pm 6.9$ (range: $35-65$ ) years, $63.3 \%$ were male, and the median vitamin D level was 17.2 (range: 2.2-57.6). The ratio of patients who had normal vitamin D levels was $18.4 \%(n=132)$, whereas it was $65 \%$ for those who had inadequate levels, $(n=467)$, and it was $16.7 \%$ for those who had vitamin D deficiency $(\mathrm{n}=120)$. The median CACS score of the patients was 0 (range: $0-3759$ ), and 59.1\% had normal calcium scores, whereas $11.3 \%$ had minimal, $16.7 \%$ had mild, $9.5 \%$ had moderate, and 3.5\% had severe calcium scores (Table 1). Demographic and laboratory findings of the patients are shown in detail in Table 1.

Table 1. Demographic and laboratory findings

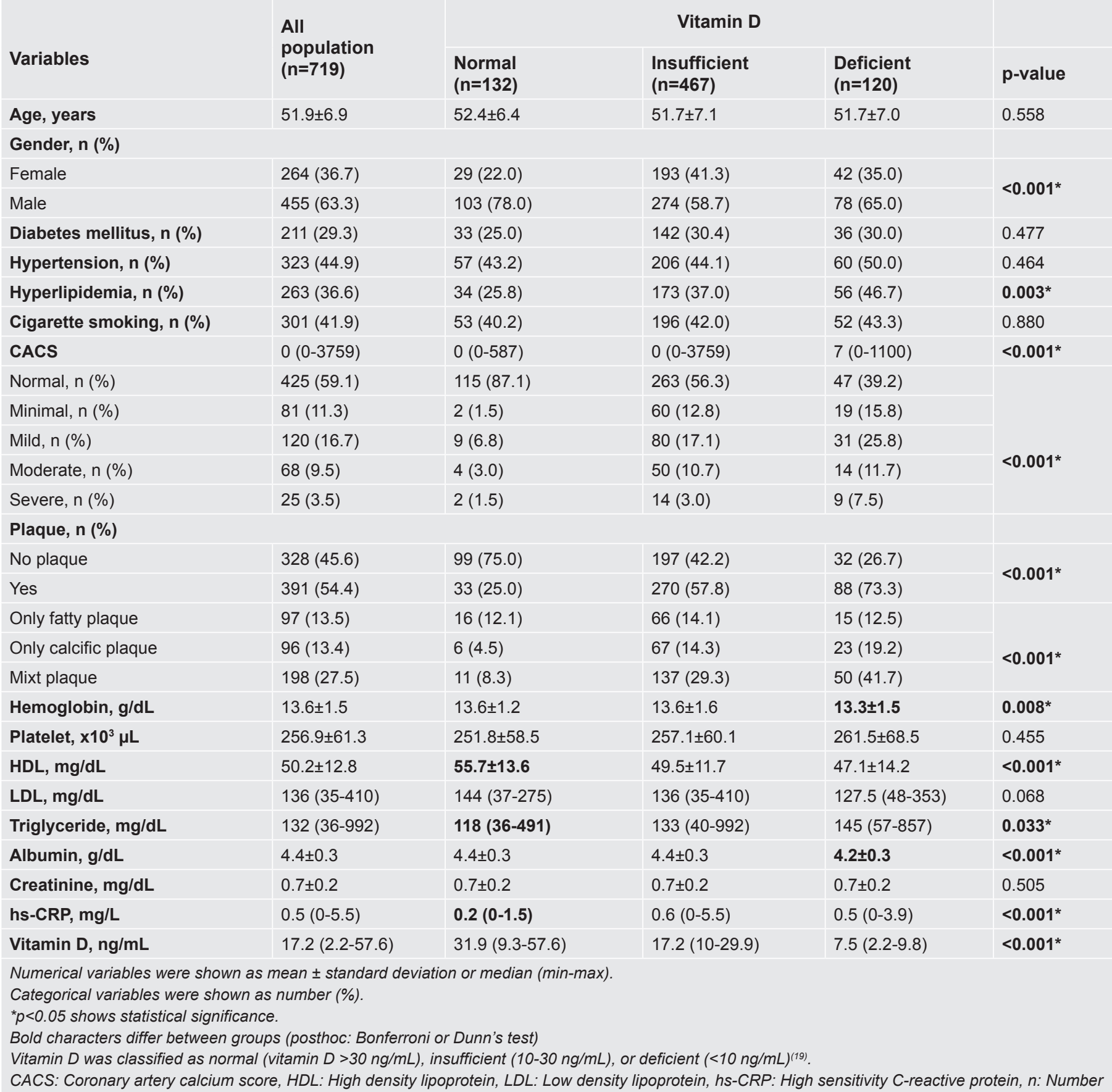


A negative correlation was detected between the vitamin D levels and CACS scores $(r=-0.345 ; \mathrm{p}<0.001)$ (Figure 1a). The median CACS in those with vitamin D deficiency was higher when compared to those with inadequate and normal levels (normal: 0 vs inadequate: 0 vs deficient 7; $\mathrm{p}<0.001)$. In terms of the plaque distribution, the ratio of those who had calcified plaque among those with vitamin D deficiency (normal: $4.5 \%$ vs inadequate: $14.3 \%$ vs deficient $19.2 \% ; \mathrm{p}<0.001)$ and the ratio of those who had mixed plaque (normal: $8.3 \%$ vs inadequate: $29.3 \%$ vs deficient $41.7 \% ; \mathrm{p}<0.001$ ) were detected higher (Figure 1b) (Table 1). In the CACS: 0 patients, it was determined that the ratio of those with a mere fatty plaque was higher in those who had vitamin D deficiency when compared to those who had inadequate or normal samples. In those who had inadequate vitamin $\mathrm{D}$, the ratio of those with

Table 2. Demographic and laboratory findings according to plaque types

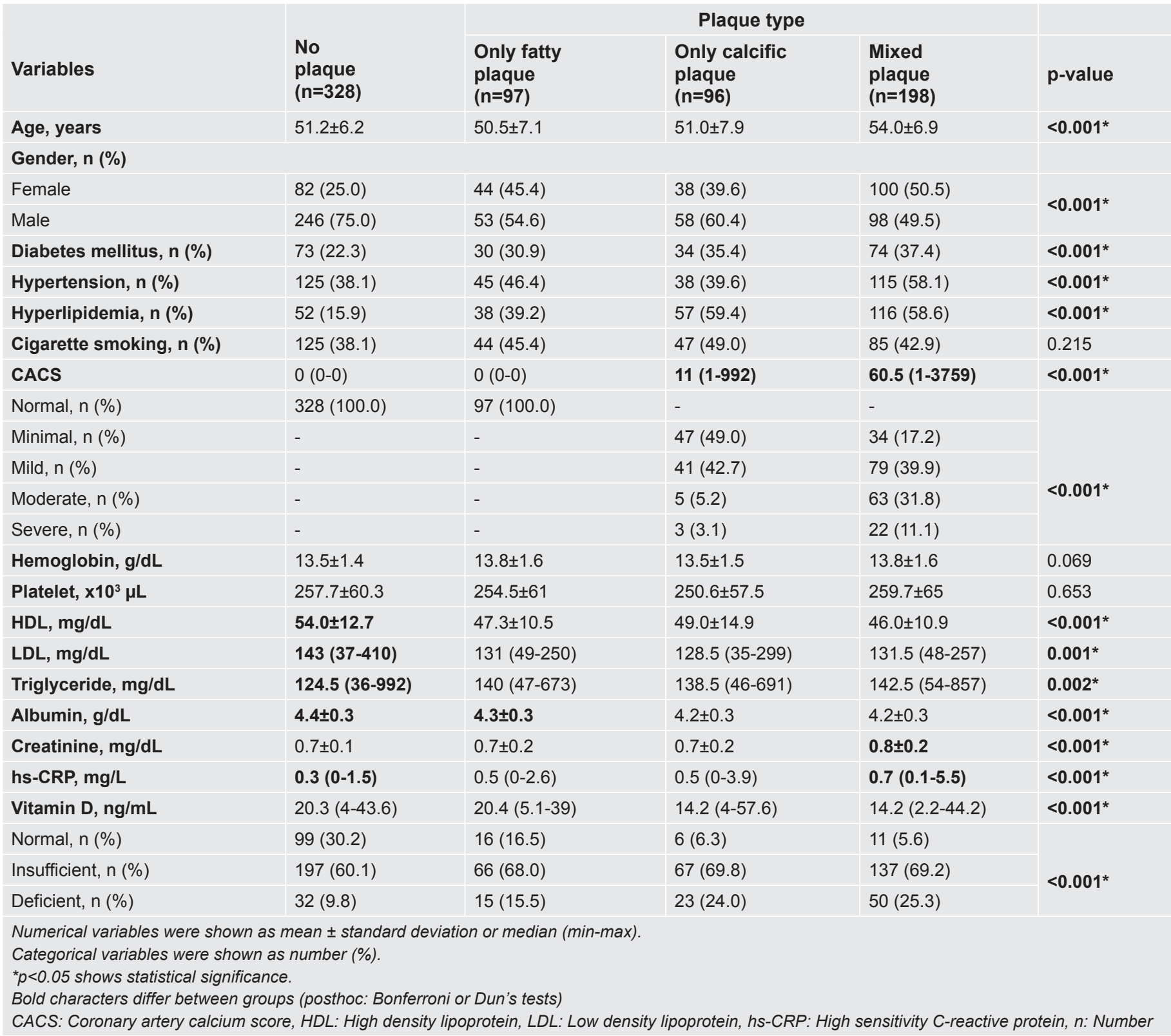


fatty plaque was found to be higher when compared to the normal ones (normal: $13.9 \%$ vs inadequate: $25.1 \%$ vs deficient: $31.9 \%$; $=0.013$ ) (Figure 1c).

The median vitamin D level was similar in those with mixed plaque and mere calcified plaque, and the vitamin D levels were lower when compared to those with mere fatty plaque and those with zero-plaque. The median vitamin D levels did not differ significantly in those with and without fatty plaque (Figure 1d and Table 2).

Compared to those with no atherosclerosis, the median CACS level and vitamin D efficiency of those with atherosclerosis $(0$ vs. $15 ; \mathrm{p}<0.001)$ were higher
(9.8\% vs $22.5 \% ; \mathrm{p}<0.001)$. The findings associated with atherosclerosis are detailed in Table 3.

Regardless of the traditional risk factors, vitamin D deficiency was found as an independent predictor for atherosclerosis (OR: 6.9; 95\% CI: 3.53-13.52; $<<0.001$ ), fatty plaque (OR: 3.04; 95\% CI: 1.34-6.87; p=0.008), mere calcified plaque (OR: 13.11; 95\% CI: 3.53-13.52; $\mathrm{p}<0.001$ ), and mixed plaque (OR: 14.27 ; 95\% CI: 5.58 $36.50 ; \mathrm{p}<0.001$ (Table 4). Moreover, regardless of the traditional risk factors, vitamin D deficiency increased the risk of fatty plaque development by 2.37 times in patients with CAC: 0 (OR: 2.37; 95\% CI: 1.01-5.62; $\mathrm{p}=0.045$ ).

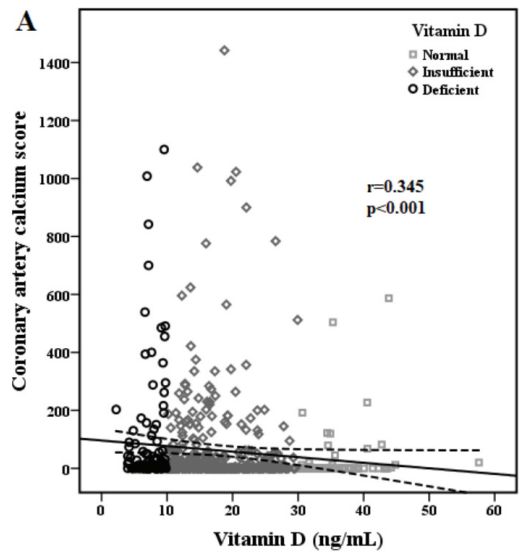

C

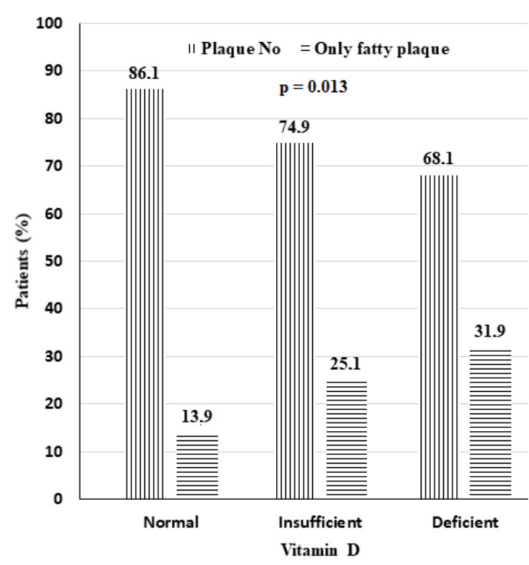

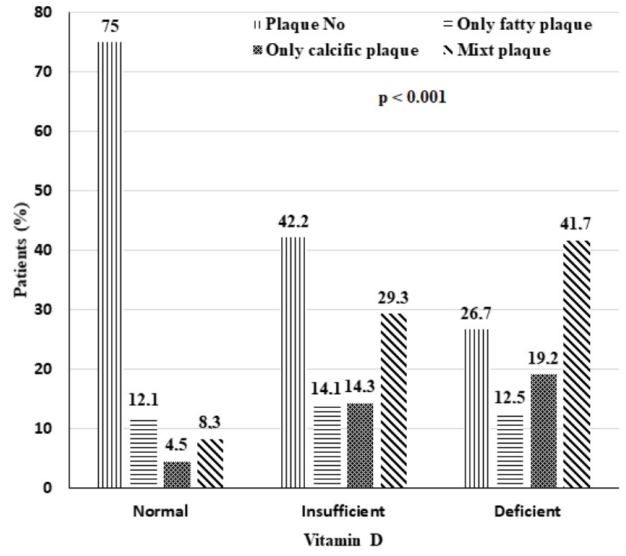

D

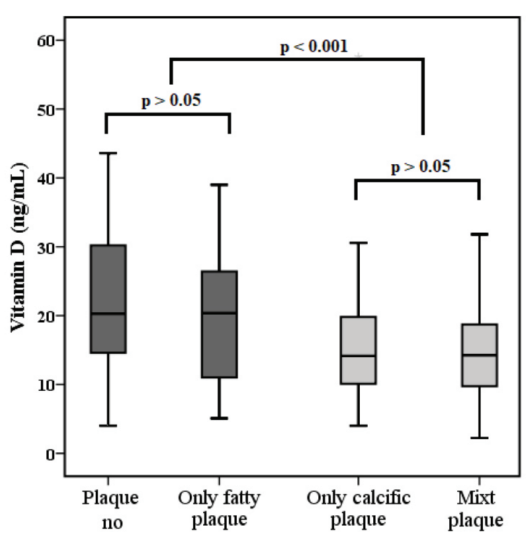

Figure 1. Vitamin D distributions: a) Relationship between coronary artery calcium score and vitamin D, b) Plaque distributions according to vitamin D sufficiency, c) vitamin D levels according to plaque distributions, d) Presence of plaque according to vitamin D adequacy in patients with coronary artery calcium score 0 
Table 3. Factors associated with atherosclerosis

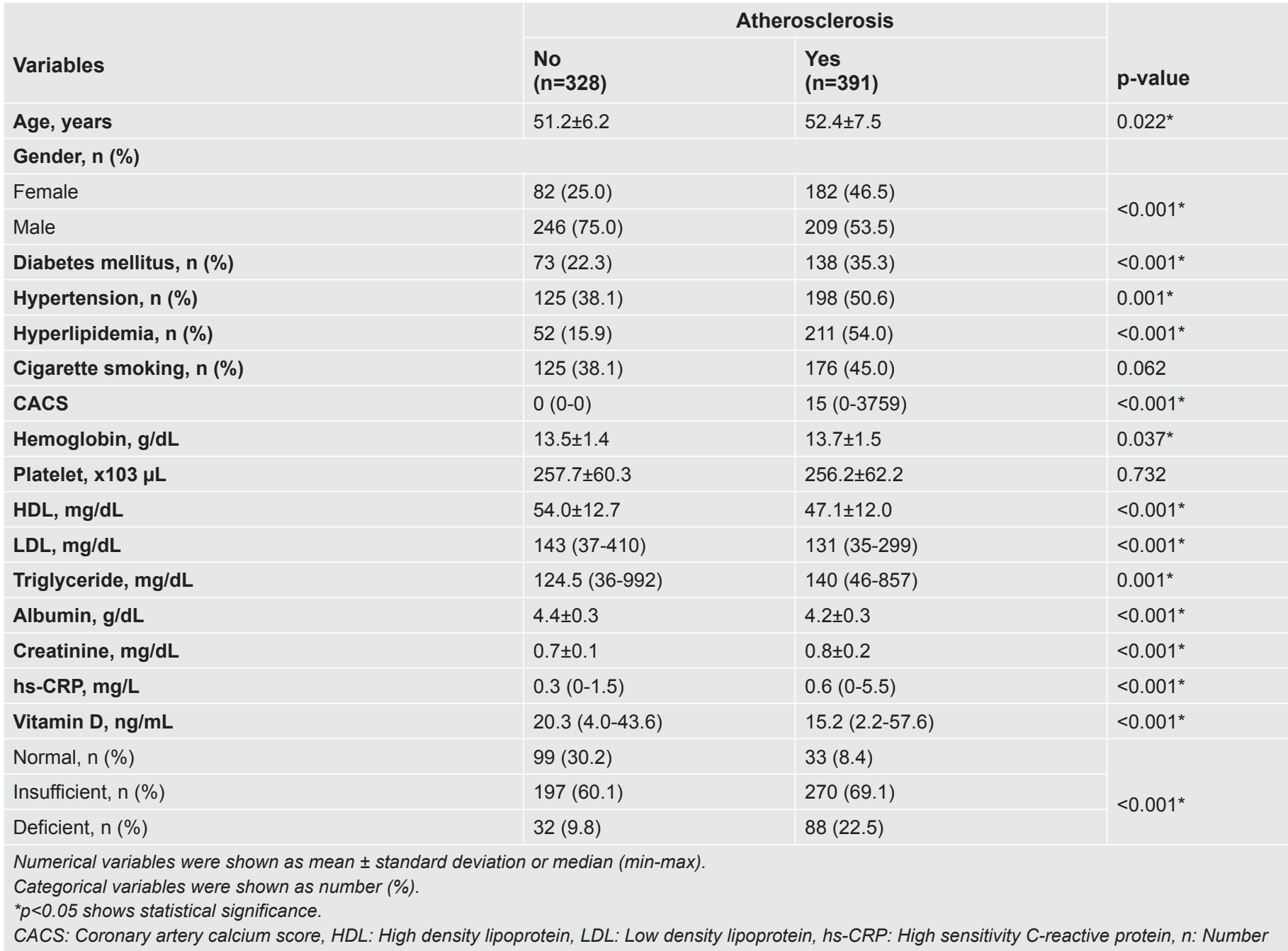

\section{Discussion}

In this study, a negative relationship was found between the CACSs and vitamin D levels, which are indicators of subclinical atherosclerosis in patients without previously known CAD. While a higher percentage of calcified and mixed plaque was detected in patients with vitamin D deficiency, vitamin D inadequacy and deficiency were identified as independent predictors of atherosclerosis. In patients with CACS: 0 , the ratio of those with a fatty plaque in vitamin D deficiency was higher and was found as the predictor of fatty plaque, regardless of traditional risk factors. These findings suggested that there may be a higher risk of CAD in asymptomatic patients with inadequate or insufficient vitamin D levels.

CAC, which is found in coronary before the development of clinically significant narrowness, is an important predictor of subclinical atherosclerosis ${ }^{(21)}$. However, vitamin D deficiency is also considered to be a potential risk factor for CAD due to its contributions to atherosclerosis ${ }^{(22,23)}$. Although vitamin D plays a role in the cardiovascular system through its pleomorphic impacts, the pathophysiology of its relationship with CAC is not fully understood; however, a number of mechanisms have been put forward. Vitamin D deficiency causes impaired calcium 
Table 4. Association of vitamin D deficiency and presence of plaque

\section{Dependent variables}

Atherosclerosis (ref: No plaque or CACS $=0$ )

OR

$95 \% \mathrm{Cl}$

p

Only fatty plaque (ref: No plaque)

OR

$95 \% \mathrm{Cl}$

p

Only calcific plaque (ref: No plaque)

OR

$95 \% \mathrm{Cl}$

p

Mixed plaque (ref: No plaque)

OR

$95 \% \mathrm{Cl}$

p

Only calcific plaque (ref: Only fatty plaque)

OR

$95 \% \mathrm{Cl}$

p

Mixed plaque (ref: Only fatty plaque)

OR

$95 \% \mathrm{Cl}$

p

Mixed plaque (ref: Only calcific plaque)

OR

$95 \% \mathrm{Cl}$

\section{p}

Age, gender, smoking, diabetes mellitus, hypertension and hyperlipidemia were adjusted in all analysis.

Those with normal vitamin $D$ levels were considered as reference.

${ }^{*} p<0.05$ shows statistical significance.

Vitamin D was classified as normal (vitamin $D>30 \mathrm{ng} / \mathrm{mL}$ ), insufficient $\left(10-30 \mathrm{ng} / \mathrm{mL}\right.$ ), or deficient $(<10 \mathrm{ng} / \mathrm{mL})^{(19)}$.

OR: odds ratio, $\mathrm{Cl}$ : confidence interval, ref: Reference

balance and secondary hyperparathyroidism. Differences in calcium and parathyroid hormone homeostasis are a predisposing factor for vascular calcification ${ }^{(24)}$. A study on pigs found that vitamin D deficiency increased the karyopherin $\alpha 4$ expression and NF- $\lambda$ B activation ${ }^{(25)}$. As a result, it was suggested that increased chronic inflammation of epicardial adipose tissue accelerates the progression of $\mathrm{CAD}^{(26)}$. It was suggested that vitamin $\mathrm{D}$ plays a role in
Nagelkerke $\mathbf{R}^{2}$

0.385

$<0.001^{*}$

0.235

0.001

0.415

$<0.001^{*}$

0.494

$<0.001^{*}$

0.272

$<0.001^{*}$

0.258

$<0.001^{*}$

0.064

$0.002^{*}$ 
the coronary calcification process by acting on antigenpresenting cells, such as dendritic cells and macrophages, by suppressing cholesterol intake ${ }^{(27)}$. However, vitamin D has an impact on all stages of atherosclerotic plaque formation, destabilization, and rupture ${ }^{(28)}$.

In the current study, a negative correlation between vitamin D and CACS was detected, but lower Vitamin D levels were determined only in patients with the calcified plaque and mixed plaque. Conflicting results have been reported in studies that researched the relationship between vitamin $\mathrm{D}$ and $\mathrm{CAC}$ in the literature ${ }^{(15-18)}$. This may have depended on research being conducted in different geographical regions. Moreover, this may have been associated with the fact that the impacts of traditional cardiovascular risk factors (age, sex, smoking status, DM, hypertension, hyperlipidemia) were not eliminated ${ }^{(29)}$. These risk factors may affect the relationship between vitamin D and CAC. In the current study, it was observed that a significant relationship continued, even in the regression model, in which the effects of these risk factors were eliminated. Furthermore, in the case of vitamin D deficiency or inadequacy, it was found that the probability of the presence of calcified and mixed plaque increased when compared to the patients with fatty plaque.

Mere non-calcified plaque was observed in $4-38 \%$ of the asymptomatic patients ${ }^{(30-32)}$. A meta-analysis showed that only $1 \%$ of patients with CACS: 0 were diagnosed with acute coronary syndrome after presenting with acute chest pain, normal troponin level, and suspected electrocardiography ${ }^{(33)}$. Moreover, it was determined in this study that CACS $>0$ had a $99 \%$ sensitivity value, $57 \%$ specificity value, $24 \%$ positive predictive value, and $99 \%$ negative predictive value for acute coronary syndrome ${ }^{(33)}$. In this research, it was observed that while the fatty plaque ratio in the whole population was $13.5 \%$, this rate increased to $25.1 \%$ in vitamin D inadequacy and $31.9 \%$ in vitamin D deficiency in patients with CACS: 0. Furthermore, it was found that vitamin D deficiency, independent of traditional risk factors, increased the likelihood of fatty plaque by about 2.4 times in this patient group. This can speed up the atherosclerotic process due to the increased presence of calcium from simple fatty plaque to mixed plaque, which is the very early stage of $\mathrm{CAD}^{(11,12)}$.

Vitamin D supplement reportedly does not change coronary artery plaque load in patients with calcified plaque $^{(12)}$, but it has been associated with improvement in cardiac results ${ }^{(23,34)}$. As far as observed in the current research, no studies that have assessed the effect of vitamin D supplements in patients with a fatty plaque in their coronary could be found. Therefore, the atherosclerotic process can be slowed down or prevented with vitamin D supplements, especially in patients with vitamin D deficiency or inadequacy. To this end, randomized controlled studies are needed.

The strengths of this study were the wide number of samples and the consideration of mixing factors. However, there were some significant restrictions. Due to fact that the study was a retrospective research, the $1.25(\mathrm{OH})_{2} \mathrm{D}$ levels of the patients could not be measured. Circulating vitamin D levels are transmitted by the VDR signal. Therefore, the measured vitamin D levels did not reflect the circulating active form of the $1.25(\mathrm{OH})_{2} \mathrm{D}$ levels. Given the complex nature of its metabolism and signal, referring to systemic levels of vitamin D alone may be insufficient to fully understand its physiological effect, especially in disease conditions. Moreover, CAC can develop over time, and the development of current calcium lesions in patients was not investigated.

\section{Conclusion}

It was found that there was a negative relationship between the vitamin D levels and the CACS, and there is a higher risk of atherosclerosis and developing calcified and mixed plaque in vitamin D deficiency. Moreover, considering the increase in the rates of fatty plaque in vitamin D deficiency in patients with a CACS of 0 , it is thought that the atherosclerotic process begins and CAD may speed up. Depending on the incidence of CVDs and vitamin D deficiency in asymptomatic patients, vitamin D supplements can be beneficial. 


\section{Ethics}

Ethics Committee Approval: İstanbul Training and Research Hospital, Clinical Research Ethics Committee (decision date/no: 24.07.2020/2481).

Informed Consent: All of the participants' consents were obtained in both verbal and written form before the study began.

Peer-review: Externally peer-reviewed.

\section{Authorship Contributions}

Surgical and Medical Practices: F.E., Y.Y., M.S.A., O.Y., A.K., Concept: F.E., M.S.A., Design: F.E., Y.Y., O.Y., A.K., Data Collection or Processing: F.E., Y.Y., M.S.A., O.Y., A.K., Analysis or Interpretation: F.E., Y.Y., M.S.A., O.Y., A.K., Literature Search: F.E., Y.Y., M.S.A., O.Y., A.K., Writing: F.E., Y.Y., M.S.A., O.Y., A.K.

Conflict of Interest: The authors declared no conflicts of interest concerning to the authorship and/or publication of this article.

Financial Disclosure: The authors declared that this study received no financial support.

\section{References}

1. Wang TJ, Pencina MJ, Booth SL, et al. Vitamin D deficiency and risk of cardiovascular disease. Circulation $2008 ; 117: 503-11$.

2. Zittermann A, Schleithoff SS, Koerfer R. Putting cardiovascular disease and vitamin D insufficiency into perspective. Br J Nutr 2005;94:483-92.

3. Dziedzic EA, Gąsior JS, Pawłowski M, et al. Vitamin D level is associated with severity of coronary artery atherosclerosis and incidence of acute coronary syndromes in non-diabetic cardiac patients. Arch Med Sci 2019;15:359-68.

4. Carbone F, Montecucco F. The role of the intraplaque vitamin d system in atherogenesis. Scientifica (Cairo) 2013;2013:620504.

5. Brewer LC, Michos ED, Reis JP. Vitamin D in atherosclerosis, vascular disease, and endothelial function. Curr Drug Targets 2011;12:54-60.

6. Chen S, Glenn DJ, Ni W, et al. Expression of the vitamin d receptor is increased in the hypertrophic heart. Hypertension 2008;52:1106-12.

7. Pilz S, Tomaschitz A, Drechsler C, Dekker JM, März W. Vitamin D deficiency and myocardial diseases. Mol Nutr Food Res 2010;54:1103-13.

8. Xiang W, Kong J, Chen S, et al. Cardiac hypertrophy in vitamin D receptor knockout mice: role of the systemic and cardiac renin-angiotensin systems. Am J Physiol Endocrinol Metab 2005;288:E125-32.
9. Budoff MJ, Gul KM. Expert review on coronary calcium. Vasc Health Risk Manag 2008;4:315-24.

10. Carr JJ, Nelson JC, Wong ND, et al. Calcified coronary artery plaque measurement with cardiac CT in population-based studies: standardized protocol of Multi-Ethnic Study of Atherosclerosis (MESA) and Coronary Artery Risk Development in Young Adults (CARDIA) study. Radiology 2005;234:35-43.

11. Doherty TM, Asotra K, Fitzpatrick LA, et al. Calcification in atherosclerosis: bone biology and chronic inflammation at the arterial crossroads. Proc Natl Acad Sci U S A 2003;100:11201-6.

12. Manson JE, Allison MA, Carr JJ, et al. Calcium/vitamin D supplementation and coronary artery calcification in the Women's Health Initiative. Menopause 2010;17:683-91.

13. Wang J, Zhou JJ, Robertson GR, Lee VW. Vitamin D in Vascular Calcification: A Double-Edged Sword? Nutrients 2018;10:652.

14. Li YC, Kong J, Wei M, Chen ZF, Liu SQ, Cao LP. 1,25-Dihydroxyvitamin $\mathrm{D}(3)$ is a negative endocrine regulator of the renin-angiotensin system. J Clin Invest 2002; 110: 229-38.

15. Malik R, Aneni EC, Roberson L, et al. Measuring coronary artery calcification: is serum vitamin D relevant? Atherosclerosis 2014;237:734-8.

16. Lichtenstein G, Perlman A, Shpitzen S, et al. Correlation between coronary artery calcification by non-cardiac CT and Framingham score in young patients. PLoS One 2018;13:e195061.

17. Lee S, Ahuja V, Masaki K, et al. A Significant Positive Association of Vitamin D Deficiency with Coronary Artery Calcification among Middleaged Men: For the ERA JUMP Study. J Am Coll Nutr 2016;35:614-20.

18. Lim S, Shin H, Kim MJ, et al. Vitamin D inadequacy is associated with significant coronary artery stenosis in a community-based elderly cohort: the Korean Longitudinal Study on Health and Aging. J Clin Endocrinol Metab 2012;97:169-78.

19. Gallagher JC, Sai AJ. Vitamin D insufficiency, deficiency, and bone health. J Clin Endocrinol Metab 2010;95:2630-3.

20. Agatston AS, Janowitz WR, Hildner FJ, Zusmer NR, Viamonte M Jr, Detrano R. Quantification of coronary artery calcium using ultrafast computed tomography. J Am Coll Cardiol 1990;15:827-32.

21. Gatto L, Prati F. Subclinical atherosclerosis: how and when to treat it? Eur Heart J Suppl 2020;22(Suppl E):E87-E90.

22. Siadat ZD, Kiani K, Sadeghi M, Shariat AS, Farajzadegan Z, Kheirmand M. Association of vitamin D deficiency and coronary artery disease with cardiovascular risk factors. J Res Med Sci 2012;17:1052-5.

23. Legarth $C$, Grimm D, Krüger $M$, Infanger $M$, Wehland $M$. Potential Beneficial Effects of Vitamin D in Coronary Artery Disease. Nutrients 2019;12:99.

24. Agarwal P, Prakash M, Singhal M, Bhadada SK, Gupta Y, Khandelwal $\mathrm{N}$. To assess vascular calcification in the patients of hypoparathyroidism using multidetector computed tomography scan. Indian J Endocrinol Metab 2015;19:785-90

25. Chen S, Swier VJ, Boosani CS, Radwan MM, Agrawal DK. Vitamin D Deficiency Accelerates Coronary Artery Disease Progression in Swine. Arterioscler Thromb Vasc Biol 2016;36:1651-9. 
26. Chen S, Swier VJ, Boosani CS, Radwan MM, Agrawal DK. Vitamin D Deficiency Accelerates Coronary Artery Disease Progression in Swine. Arterioscler Thromb Vasc Biol 2016;36:1651-9.

27. Young KA, Snell-Bergeon JK, Naik RG, et al. Vitamin D deficiency and coronary artery calcification in subjects with type 1 diabetes. Diabetes Care 2011;34:454-8

28. Kassi E, Adamopoulos C, Basdra EK, Papavassiliou AG. Role of vitamin D in atherosclerosis. Circulation 2013;128:2517-31.

29. Berry JD, Lloyd-Jones DM, Garside DB, Greenland P. Framingham risk score and prediction of coronary heart disease death in young men. Am Heart J 2007;154:80-6.

30. Li M, Sun G, Ding J, Li L, Peng ZH, Jiang XS. Risk factors for non-calcified plaques in asymptomatic population. Acad Radiol 2012;19:548-53.
31. Iwasaki K, Matsumoto T, Aono H, Furukawa H, Samukawa M. Prevalence of non-calcified coronary plaque on 64-slice computed tomography in asymptomatic patients with zero and low coronary artery calcium. Can J Cardiol 2010;26:377-80.

32. Choi EK, Choi SI, Rivera JJ, et al. Coronary computed tomography angiography as a screening tool for the detection of occult coronary artery disease in asymptomatic individuals. J Am Coll Cardiol 2008;52:357-65.

33. Criqui MH, Denenberg JO, Ix JH, et al. Calcium density of coronary artery plaque and risk of incident cardiovascular events. JAMA 2014;311:271-8.

34. Bahrami LS, Ranjbar G, Norouzy A, Arabi SM. Vitamin D supplementation effects on the clinical outcomes of patients with coronary artery disease: a systematic review and meta-analysis. Sci Rep 2020;10:12923. 